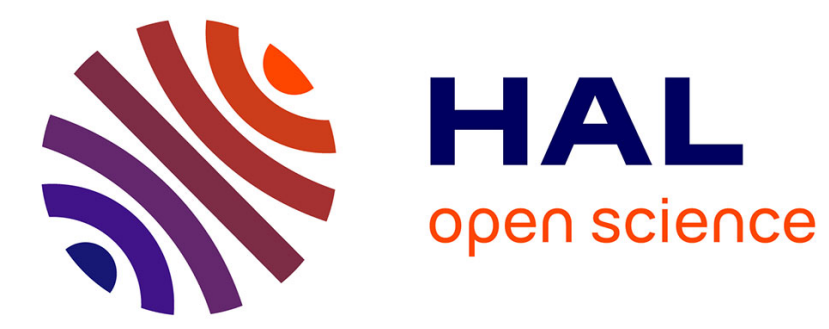

\title{
Le cinéma, instrument de politique extérieure du fascisme italien
}

Christel Taillibert

\section{To cite this version:}

Christel Taillibert. Le cinéma, instrument de politique extérieure du fascisme italien. Mélanges de l'École française de Rome - Italie et Méditerranée, 1998, 10.3406/mefr.1998.4595 halshs-02192642

\section{HAL Id: halshs-02192642 \\ https://shs.hal.science/halshs-02192642}

Submitted on 24 Jul 2019

HAL is a multi-disciplinary open access archive for the deposit and dissemination of scientific research documents, whether they are published or not. The documents may come from teaching and research institutions in France or abroad, or from public or private research centers.
L'archive ouverte pluridisciplinaire HAL, est destinée au dépôt et à la diffusion de documents scientifiques de niveau recherche, publiés ou non, émanant des établissements d'enseignement et de recherche français ou étrangers, des laboratoires publics ou privés. 


\section{Christel Taillibert}

\section{LE CINÉMA, INSTRUMENT DE POLITIQUE EXTÉRIEURE DU FASCISME ITALIEN}

Introduction : la politique extérieure fasciste.

Quand il prit le pouvoir en Italie en 1922, Benito Mussolini s'attacha à développer une politique extérieure rassurante afin de tranquilliser ses voisins européens quant à ses intentions. Dans l'Europe encore brûlante des carnages de la première guerre mondiale, la prise du pouvoir par un mouvement se réclamant du fascisme au cœur même de l'Europe ne pouvait laisser indifférentes les démocraties occidentales. Le nouveau Chef d'État italien chercha donc à satisfaire les jeux diplomatiques de l'après-guerre et à inscrire sa politique extérieure dans le puzzle compliqué des traités et des accords qui marqua les années vingt ${ }^{1}$.

Au-delà des stratégies diplomatiques italiennes, le fascisme italien était tout entier construit sur un programme nationaliste : la volonté de "redressement national" chère au Duce entendait rendre à l'Italie la place qu'elle méritait en Europe, et ce faisant lui permettre de retrouver la considération dont elle avait bénéficié au cours de l'histoire de la part des autres nations.

Si ce projet s'appuyait tout particulièrement sur une forte idéologie colonialiste, Mussolini masqua plus ou moins 
jusqu'en 1935 ses intentions de conquêtes territoriales et fit preuve au contraire de sa volonté de collaborer activement à la construction de la Société des Nations. Il cherchait ainsi à démontrer les bonnes intentions internationales du fascisme italien et sa volonté d'agir concrètement en faveur de la paix. Peu à peu, ce soucis de respectabilité porta ses fruits et le fascisme italien se construisit aux yeux des démocraties une image honorable bien souvent admirée à l'étranger. Il est vrai que le gouvernement fasciste pouvait se prévaloir d'un certain nombre de succès de politique intérieure - au niveau économique, financier, social et culturel - qu'il exploitait au maximum pour alimenter sa propagande extérieure.

Dans ce contexte déjà favorable, la crise économique mondiale déclenchée en 1929 par le krach boursier de Wall Street provoqua un véritable mouvement en faveur du fascisme. Alors que la confiance envers le modèle démocratique libéral était fortement ébranlée, le fascisme apparaissait comme une alternative politique possible. Cette considération internationale fut évidemment mise à profit par Benito Mussolini qui renforça son œuvre de propagande en faveur des réalisations de son Gouvernement.

Dans l'édifice complexe de cette promotion du fascisme à l'étranger, la sphère culturelle n'était point absente des préoccupations du régime. Le Duce avait en effet compris combien la domination intellectuelle et artistique était importante pour conférer à l'Italie le prestige international auquel elle aspirait ${ }^{2}$. C'est à la place qu'occupait le cinéma 
dans cette œuvre de propagande internationale que nous allons nous intéresser dans cet article. Mussolini comprit en effet très tôt le rôle qu'était appelé à jouer ce moyen de communication, encore jeune à l'époque, et l'importance qu'il y avait pour son pays de se montrer à la pointe dans ce domaine.

Pour illustrer ce phénomène, nous nous intéresserons principalement dans cet article au secteur du cinéma d'éducation et d'enseignement, et ceci à travers deux institutions - l'Institut National LUCE puis l'Institut International du cinématographe éducatif . Ce phénomène fut en effet beaucoup plus significatif dans le domaine du cinéma de non-fiction, même si l'industrie du film spectaculaire fut elle aussi investie, mais plus tard dans l'histoire du fascisme italien.

\section{L'Institut National LUCE.}

Au début de l'année 1924 naquit une petite société anonyme sous le nom de "Sindacato Istruzione Cinematografica"3, plus communément appelée "S.I.C." Le but de cette entreprise était de développer l'usage du cinématographe en tant qu'auxiliaire didactique dans les écoles italiennes. Cette petite société qui occupait un modeste local à Rome, le long du Tibre, fut rapidement confrontée à de sévères problèmes financiers. Luciano de Feo, concepteur de ce projet et Directeur de la jeune institution, ne put trouver 
aucun appui financier auprès des administrations concernées par les problèmes de l'éducation. Pour rendre viable sa société, il savait que la seule solution était d'intéresser le Gouvernement italien à cette affaire afin qu'il lui apportât son concours financier. Pour ce faire, il devait donc convaincre Benito Mussolini du rôle fondamental que pouvait jouer le cinématographe en faveur de l'éducation des masses. Cette préoccupation revêtait une dimension particulière au regard des nouveaux idéaux que le régime fasciste entendait transmettre au peuple italien.

En avril 1924, Luciano de Feo rencontra Giacomo Paulucci di Calboli - alors Chef de Cabinet du Ministre des Affaires étrangères - qui était aussi un de ses amis puisqu'ils avaient fait leurs études d'économie ensemble puis écrit dans les colonnes du journal La Perseveranza, journal milanais d'obédience fasciste, à la fin des années dix. Il eut ainsi la possibilité de lui exposer son programme en matière de cinématographie éducative. Ce dernier envisagea immédiatement la possibilité de lui donner plus d'ampleur et en référa au Duce. Celui-ci donna son feu vert à la participation du Gouvernement italien à cette entreprise, et c'est ainsi qu'en juillet 1924, le S.I.C. changea de nom et prit celui de "L.U.C.E.", sigle choisi par Benito Mussolini lui-même et qui signifiait "L'Unione Cinematografica Educativa"4 . La petite société anonyme devint donc une organisation étatique à part entière mise au service du régime fasciste. Giuseppe de Michelis en devint Président, Giacomo Paulucci di Calboli VicePrésident et Luciano de Feo Directeur-Général 5 . 
$\mathrm{Au}$ cours des années qui suivirent, l'Institut National LUCE connut un développement impressionnant. Il bénéficia en particulier du décret que fit voter Benito Mussolini le 3 avril 1926, lequel rendait obligatoire la projection dans toutes les salles de cinéma du Royaume d'Italie des films produits par cet Institut.

A l'étranger, cette expérience italienne - la première en son genre - ne manqua pas de susciter l'admiration des éducateurs qui, dans leur propre pays, se battaient corps et âme pour développer l'usage du cinéma dans une perspective éducative et didactique mais ne rencontraient que de l'incompréhension auprès de leurs Gouvernements respectifs.

Les débats qui eurent lieu à Bâle en avril 1927 à l'occasion de la Conférence Européenne du film d'enseignement étaient particulièrement significatifs à ce sujet. L'Institut National LUCE y était représenté par trois délégués dont Luciano de Feo lui-même. Cet organisme fut l'objet d'une reconnaissance officielle de la part des congressistes de tous pays qui participaient à cette manifestation : en effet, trois des résolutions adoptées à cette occasion applaudissaient les réalisations italiennes en faveur du développement du cinéma d'éducation et d'enseignement.

La première d'entre elles proposait à toutes les Nations du monde de prendre l'Italie et son "Istituto Nazionale LUCE" pour exemple et modèle. On espérait ainsi que d'autres pays 
suivissent le même chemin et que leurs Gouvernements créassent des bureaux et instituts à caractère national, condition que l'on estimait indispensable au progrès général de la cinématographie d'éducation et d'enseignement.

Cette mise en exergue de la situation italienne flatta beaucoup les ressortissants de ce pays, lesquels ne manquèrent pas de rendre compte à leur Gouvernement de cette heureuse distinction. Ce dernier prit rapidement la mesure du prestige national qui pouvait lui revenir s'il donnait à cette position de leader international dans ce domaine les moyens de s'affirmer. Voici l'extrait d'un rapport rétrospectif rédigé par G. Michel Coissac - le Directeur de la revue Le Cinéopse - à propos de ce Congrès. Ces lignes étaient particulièrement éloquentes à propos du crédit dont bénéficia à cette occasion la délégation italienne :

“En 1927, à Bâle, au Congrès international du film scolaire, le représentant de l'Italie, M. Luciano de Feo avait guidé les discussions sur un terrain pratique en ce qui concernait le film d'enseignement et démontrait comment, au lendemain de la guerre, il était nécessaire d'envisager des réformes scolaires, radicales et profondes en vue de l'éducation des nouvelles générations et comment il fallait ajouter à ces réformes la question du changement de méthode afin que soit permise l'introduction systématique du film dans l'enseignement. Et la conclusion de ces discussions était documentée dans une résolution où l'exemple pratique de l'Italie était donné à tous les pays du monde" . 
Une seconde résolution votée au Congrès de Bâle désignait en conséquence directe de la première Rome en tant que siège de la prochaine Conférence Européenne consacrée à la cinématographie d'enseignement. Voici le commentaire du Français Jehan de Vimbelle à propos de ce choix :

"Le choix de Rome est intéressant. En effet, M. Mussolini est un fervent du cinéma éducatif, et l'on sait que, lorsque le "Duce" épouse une idée, il ne recule devant rien pour la réaliser jusqu'au bout. C'est ainsi que, à son initiative, s'est créée à Rome une vaste "Centrale", la "LUCE", encore en pleine voie d'organisation, mais qui est conçue sur un plan si grandiose qu'on peut le craindre un peu... théorique"7.

On assista donc à cette date à une véritable modification des forces nationales en matière de cinématographie éducative : alors que jusque-là, les délégations allemandes et françaises dominaient largement les débats, l'Italie se présentait dorénavant comme le nouveau leader en la matière.

La troisième des résolutions votées à cette occasion stipulait que Luciano de Feo serait Président du "Comité permanent d'action" dont on décida alors la constitution et auquel on confia la charge de poursuivre les travaux entamés un an plus tôt au Congrès international de cinématographie de Paris : il s'agissait de constituer dans le cadre de la Société des Nations un organisme international consacré à la cinématographie éducative, projet qui se concrétisera avec la 
constitution de l'Institut International du cinématographe éducatif, dont nous aurons l'occasion de parler plus avant.

L'admiration dont fut l'objet l'Institut National LUCE à cette occasion permit au Gouvernement italien de comprendre le rôle que pouvait jouer la cinématographie éducative dans l'œuvre de propagande internationale fasciste. Il eut en effet la satisfaction d'être considéré comme un modèle à l'étranger : au cours des années vingt, de nombreux Gouvernements suivirent son exemple et ouvrirent des organismes analogues à l'Institut romain. En Grande-Bretagne par exemple fut constitué le "British Film Institute". Voici de quelle façon son Vice-Président, Richard S. Lambert, retraçait la création de l'organisme italien et vantait la réalisation du Gouvernement fasciste :

“On était alors en 1924. Les efforts tenaces de quelques individus isolés étaient méconnus. Seules, quelques très rares personnalités politiques avaient, jusqu'alors, parlé du cinéma comme moyen d'éducation et d'instruction des masses, mais il ne s'était pas trouvé un gouvernement pour entrer résolument au cœur de la question, et affirmer, par un acte législatif, que le cinéma est un des plus nobles et des plus puissants instruments d'élévation intellectuelle des masses. Acte de volonté qui aurait dû - les faits l'ont démontré par la suite servir d'exemple à d'autre pays"'.

Ainsi, un peu partout, on flattait la clairvoyance du régime fasciste qui le premier avait su allouer de véritables 
moyens au développement de la cinématographie éducative. Luciano de Feo lui-même ne se lassait de rappeler au Chef de Gouvernement le prestige que lui rapportait la primauté de l'Italie en la matière et la nécessité qu'il y avait de la conserver. Cet extrait d'un article qu'il publia en 1936 était particulièrement clair à ce sujet :

"Mais une chose est certaine. Que le régime fasciste sut le premier, et grâce à l'œuvre de son Chef, comprendre et percevoir clairement ce que pouvait signifier pour son peuple l'arme magnifique et terrible de l'écran, apte à créer et à forger les esprits, ou à les déprimer, à volonté, selon la mentalité de celui qui entend l'utiliser et selon que l'on veuille donner à l'écran un habit de pur mercantilisme ou un sens de haute spiritualité. De nombreux autres Instituts du genre furent créés dans le monde. Après, pas avant. L'Institut "LUCE" a été une lumière pour tous"

La conséquence logique de ce processus fut la décision de Benito Mussolini d'assurer son pays la préservation de cette suprématie qu'il avait su conquérir en matière de cinématographie éducative et qui lui valait tant de considération à l'étranger.

\section{L'Institut International du cinématographe éducatif.}

Quelques mois seulement après le Congrès de Bâle, le Gouvernement Royal d'Italie proposa officiellement la 
constitution d'un grand "Institut International du cinématographe éducatif", soumis à l'autorité de la Société des Nations mais qu'il s'engageait à financer entièrement.

Ce projet était en discussion dans les différentes assemblées internationales depuis le Congrès international de cinématographie de Paris en 1926, mais il semblait impossible de passer à sa réalisation du fait de l'absence de crédits. La proposition du Gouvernement Italien, énoncée pour la première fois le 6 septembre 1927 par le sénateur Cippico devant l'Assemblée de la Société des Nations, était donc fort alléchante. Voici un extrait du discours que ce dernier prononça à cette occasion :

“Le Gouvernement royal d'Italie, en considération des vœux formulés par plusieurs réunions et congrès internationaux, encouragé par de nombreuses expériences faites en Italie sur l'application du cinématographe à l'éducation intellectuelle de la nation et sur son utilisation comme système auxiliaire d'enseignement, dans toutes les catégories d'écoles publiques (...) a décidé de proposer la création d'un Institut International du cinématographe éducatif, qui serait établi à Rome et qui, suivant l'article 24 du Pacte, serait placé sous la direction de la Société des Nations. A cet effet, le Gouvernement royal d'Italie offre, outre les fonds nécessaires pour le premier établissement de l'Institut ainsi que pour sa gestion normale, le siège même de cette organisation" ${ }^{\prime 10}$. 
La décision du Gouvernement italien de financer l'Institut International du cinématographe éducatif ne peut être comprise que dans la continuité de l'œuvre initiée avec la création de l'Institut National LUCE. Ce nouvel organisme ne présentait en effet comme un moyen de faire connaître au monde entier l'action développée en Italie en faveur du film d'enseignement et d'éducation.

L'Institut National LUCE joua d'ailleurs un rôle actif dans la création de l'Institut International du cinématographe éducatif : son Président, Filippo Cremonesi, participa aux travaux relatifs à la rédaction d'un schéma de statuts pour le futur organisme. Voici un extrait d'une lettre que ce dernier envoya au Ministre des Affaires étrangères le 30 juin 1927 et dans laquelle les ambitions du Gouvernement par rapport au futur Institut de Rome étaient particulièrement claires :

"La LUCE est heureuse et fière de pouvoir collaborer avec toutes ses forces à la victorieuse affirmation de Notre Pays dans le domaine de la cinématographie éducative. N'hésitons pas à déclarer que l'institution que Votre Excellence est en train d'étudier et entend réaliser pourra être d'un grand avantage pour notre Pays"11.

La liaison directe entre les deux organismes était aussi incarnée par la présence à la Direction de l'Institut de Rome de Luciano de Feo, l'ancien Directeur-Général de l'Institut National LUCE. Il était donc entendu que celui-ci poursuivrait 
au sein de ce nouvel organisme le projet général italien en matière de cinématographie éducative.

Les travaux préparatoires à la mise en place de l'Institut International du cinématographe éducatif furent relativement rapides puisque moins d'une année s'écoula avant l'adoption de son Statut organique par le Conseil de la Société des Nations, le 30 août 1928. Ce zèle s'expliquait principalement par l'investissement personnel dont fit preuve Benito Mussolini dans la genèse de cet organisme. On retrouve ainsi trace de nombreuses lettres écrites de sa main et dans lesquelles il négociait l'avancement de ce projet. Le Chef du Gouvernement italien fit d'ailleurs l'honneur de sa présence à l'occasion de l'inauguration du nouvel Institut, laquelle survint le 5 novembre 1928 à la Villa Falconieri de Frascati, l'un des deux bâtiments que lui offrait le Gouvernement pour développer ses activités. Le second siège de l'Institut International du cinématographe éducatif était lui aussi particulièrement significatif de la sollicitude que lui accordait le Duce : il s'agissait en effet d'une bâtisse médiévale située dans les jardins mêmes de la Villa Torlonia de Rome, celle-là même où Benito Mussolini et sa famille avaient élu domicile.

Celui que l'on appelait un peu partout dans le monde "l'Institut de Rome" développa une activité frénétique en faveur de la plus grande utilisation du cinéma à des fins d'éducation et d'enseignement. Il réalisa la synthèse de l'ensemble de la recherche mondiale en la matière, lança des enquêtes de portée internationale, publia une revue mensuelle 
en cinq langues qui diffusait sa pensée à travers le monde, mit en place de nombreuses actions pratiques destinées à améliorer la condition du film éducatif et d'enseignement, suscita la création d'un réseau d'Instituts Nationaux qui travaillait dans chaque pays sous le patronage de l'organisme central de Rome, etc. ${ }^{12}$.

Si l'on tente d'établir un bilan des travaux accomplis par l'Institut International du cinématographe éducatif au cours de ses dix ans d'existence, il est indéniable que ce petit organisme qui ne disposait que de faibles moyens humains et financiers réalisa une œuvre impressionnante. Ceci explique qu'il s'entoura très rapidement d'une renommée considérable. Voici à titre d'exemple un commentaire dû à M. Dufour-Féronce, sous-secrétaire général de la Société des Nations, un an et demi seulement après l'inauguration de l'organisme en question :

“Lorsque - il n'y a pas encore deux ans de cela - cet Institut fut fondé grâce à la munificence du Gouvernement de Votre Majesté, il existait déjà, dans plusieurs pays, des Institutions ou des organisations privées s'occupant du film éducatif et de sa production. Mais il était réservé à l'Institut International du cinématographe éducatif de réveiller pleinement l'intérêt pour le film éducatif, de faire comprendre tout ce que le film peut donner sur le terrain de l'enseignement, de l'éducation et de la culture générale. (...)

Grâce à ce labeur, l'Institut, qui est placé sous l'autorité de la Société des Nations, est désormais devenu, dans le sens 
international, le centre de tout ceux qui reconnaissent à quelle fin utile et instructive le film peut servir" 13 .

Les demandes d'information qui arrivaient à l'Institut de Rome constituaient une preuve quotidienne de la reconnaissance de ses travaux au niveau international. Il bénéficiait effectivement d'une image très positive dans le monde, pour l'entier bénéfice du Gouvernement qui avait pris l'initiative de le constituer et qui en assurait le financement. Il remplissait donc parfaitement son rôle et satisfaisait la volonté de reconnaissance internationale italienne, volonté qui attendait beaucoup de la sphère culturelle laquelle rendait compte du niveau d'élévation spirituelle général de la nation. Les dirigeants de l'Institut ne manquaient pas de faire valoir à leur Gouvernement toutes les manifestations de cette admiration dont était l'objet l'organisme romain de par le monde. Ainsi, dès septembre 1929, soit moins d'un an après son inauguration, Luciano de Feo envoyait-il le message suivant à Benito Mussolini :

“Sachant que cela fera plaisir à Votre Excellence, j'ai l'honneur de Lui communiquer que, au cours du mois d'août, les articles et les communications publiés en faveur de l'Institut, dans tous les pays du monde, dépassent le millier” ${ }^{14}$.

Ainsi, la vocation première de l'Institut de Rome consistant à asseoir la primauté mondiale de l'Italie en matière de cinématographie éducative ne fut jamais perdue de vue par ses dirigeants. Ces derniers savaient d'ailleurs que ce n'était 
qu'à cette condition qu'ils conserveraient l'appui du Gouvernement italien. A l'occasion du troisième anniversaire de l'Institut, Luciano de Feo envoya une lettre au Duce dans laquelle il lui donnait une fois encore l'assurance de l'énergie qu'il dépensait à le satisfaire. On pouvait en effet dans cette missive lire les lignes suivantes :

"Je peux garantir à Votre Excellence que rien ne sera négligé et que tout sera fait pour que l'Institut - aujourd'hui connu et apprécié dans le monde entier - puisse développer une œuvre toujours plus grande, digne de la Patrie qui nous commande d'affirmer en chaque instant - n'importe où et contre tous - sa volonté de toujours être le premier"15.

La publication mensuelle de l'Institut, la Revue Internationale du cinéma éducateur, contribuait d'une manière étonnante à promouvoir l'action de l'organisme romain à travers le monde. Publiée en cinq langues, elle était en effet diffusée dans de très nombreux pays dans lesquels elle était particulièrement appréciée. Des messages d'encouragement parvenaient en effet régulièrement à Rome et témoignaient du bon accueil de cette publication à l'étranger. Luciano de Feo était totalement conscient de la fonction de la Revue pour consolider le prestige de son Institut dans le monde, comme en témoignait la suite de la lettre datant de septembre 1929 que nous avons précédemment évoquée :

"Je promets à Votre Excellence que la Revue atteindra le tirage mensuel de trente mille exemplaires diffusés dans cinquante- 
deux pays. De cette façon, le mot d'ordre qui me fut donné par Votre Excellence de créer à Rome, dans l'Italie "fascistissime", un centre mondial d'études de tous les problèmes (....) du cinéma sera réalisé avec la joie immense de toujours servir (...) Votre Excellence et le Fascisme"16.

De la même façon, au cours de ses dix ans d'existence, la totalité de l'activité de l'Institut de Rome pourrait être caractérisée par cette volonté de concilier sa double identité. En effet, la fonction ouvertement reconnue de cet organisme en tant qu'élément de propagande fasciste était en parfaite opposition avec les fondements idéologiques de son activité. En effet, de par son appartenance et sa dépendance envers la Société des Nations, l'Institut International du cinématographe éducatif était un produit de l'esprit internationaliste qui caractérisa l'entre-deux guerres et dont l'institution genevoise était l'incarnation. Les écrits publiés par les rédacteurs de l'Institut étaient toujours emprunts de cette utopie qui caractérisa les lendemains de la première guerre mondiale. Luciano de Feo lui même était convaincu des possibilités du cinématographe pour forger cette ère de paix universelle, abolir les préjugés que les races entretenaient les unes sur les autres, et élever l'esprit des masses à des considérations supérieures.

Les dirigeants de l'Institut de Rome réussirent ainsi au cours des années un véritable tour de force consistant à permettre à toutes leurs réalisations au service des ambitions fascistes. Le Festival cinématographique de Venise est 
particulièrement significatif en ce sens. En effet, bien que sa contribution soit aujourd'hui complètement méconnue, c'est l'Institut International du cinématographe éducatif qui non seulement conçut le principe de cette manifestation mais permit la réalisation pratique des premières éditions. L'objectif de Luciano de Feo par rapport à ce festival était de faire connaître et de promouvoir le meilleur de la cinématographie mondiale, espérant ainsi que l'élite culturelle acceptât le film en tant que véritable manifestation artistique. Le premier Festival de 1932 fut un véritable succès, ce qui décida ses promoteurs de renouveler l'expérience dans le cadre de la Biennale suivante. Les éditions de 1934 et de 1935 furent elles aussi des réussites totales. Le Gouvernement italien commença seulement alors à entendre le discours de l'Institut de Rome, selon lequel les fêtes cinématographiques vénitiennes contribuaient énormément à l'œuvre de propagande fasciste à l'étranger. Dès la première édition de 1932, Charles Delac - le Président de la Chambre française de l'industrie cinématographique - prononça un discours dans lequel il ne pouvait être plus clair quant à ce phénomène :

"L'orateur salue donc notre Gouvernement National et le Duce qui ont compris ce problème [le cinéma], et l'Italie, qui offrit les premiers films véritablement artistiques, tels que l'inoubliable Cabiria de D'Annunzio, et apporte aujourd'hui une magnifique contribution à cet art qui en sortira fortifié des rencontres vénitiennes. L'orateur conclut : "Je présente mon hommage respectueux à Son Excellence Benito Mussolini 
qui est considéré par nous, Français, à l'égal de César et Napoléon"17.

Ayant pris conscience de ce phénomène, le Gouvernement italien décida en 1935 d'annualiser ce festival et de le placer sous la coupe du Ministère de la Presse et de la propagande.

Le Congrès international de la cinématographie d'enseignement et d'éducation organisé à Rome en 1934 témoignait lui aussi parfaitement de ce soucis constant des dirigeants de l'organisme romain de contenter le Gouvernement qui le faisait vivre. Cette énorme manifestation eut lieu du 19 au 25 avril 1934 au sein de la Villa Torlonia et rassembla 419 congressistes représentant officiellement ou officieusement 45 pays différents. Inaugurée au Capitole par Benito Mussolini lui-même, ce Congrès participa activement à satisfaire les besoins de la propagande fasciste. En effet, ce rassemblement signa définitivement l'attribution à l'Institut de Rome de la première place mondiale en matière de cinématographie éducative. $\mathrm{Au}$ cours de la période préparatoire, Benito Mussolini rédigea une circulaire qui fut distribuée aux diverses ambassades italiennes dans le monde afin de les inciter à faire activement de la publicité pour cette manifestation. Ce texte, dont voici un extrait, était particulièrement clair quant au profit qu'il espérait retirer de ces rencontres : 
"L'initiative a le plein appui du Gouvernement qui voit avec intérêt à quel point elle peut contribuer à accroître le prestige et l'importance de l'Institut, à élargir sa sphère d'action dans un domaine pour lequel l'Italie a la première indiqué une voie constructive et dans lequel il a réalisé des progrès notables. C'est donc de l'intérêt du Gouvernement que le Congrès impulsé par l'Institut International du cinématographe éducatif constitue un plein succès quant au nombre et à l'importance des délégations et des institutions représentées"18.

Le Duce ne manqua pas de profiter de la présence à Rome de ces nombreux ressortissants étrangers pour mettre en valeur les réalisations fascistes. Par exemple, en marge des débats, les congressistes furent invités à visiter l'Exposition de la Révolution fasciste célébrant le dixième anniversaire de la prise du pouvoir par les fascistes en Italie. On organisa de même à leur intention des excursions à Littorio et à Sabaudia, deux villes nouvelles nées de l'œuvre de "bonification intégrale" chère au Duce.

Nous n'avons proposé ici que quelques exemples parmi les plus probants, mais l'activité toute entière de l'Institut International du cinématographe éducatif était fondée sur ces contradictions permanentes, lesquelles rendaient parfaitement compte des contradictions sur lesquelles fonctionnait le régime fasciste italien tout entier : d'une part, une politique intérieure basée sur un discours nationaliste et colonialiste, et d'autre part, une politique extérieure qui s'affichait comme 
soumise aux principes de la Société des Nations et désireuse de contribuer à son avènement.

La position de l'Institut de Rome est fort intéressante dans la mesure ou pour démontrer sa soi-disant volonté de travailler en faveur de la coopération internationale, une des principales voies empruntées par le Gouvernement italien était la mise en avant des organismes qu'il avait créés et qu'il finançait au bénéfice de la Société des Nations : l'Institut International pour l'unification du droit privé en 1926, puis l'Institut International du cinématographe éducatif deux ans plus tard. Benito Mussolini exploitait au maximum ces initiatives afin d'assurer la communauté internationale de ses bonnes intentions en matière de politique extérieure. Comme il le disait devant la Chambre des Sénateurs à propos de la constitution de ces organismes internationaux, "ce sont des “faits" plus éloquents que n'importe quel discours pour caractériser l'attitude du Gouvernement fasciste envers la Société des Nations"19.

Dans un article qu'il publia en juillet 1929, Louis-Dop rendait compte de l'appréciation à l'étranger de ces gestes dont était redevable la Société des Nations envers le Chef du Gouvernement italien. Voici en effet ce qu'il écrivait :

"Ces deux dernières Institutions placées directement sous l'autorité et les directives du Conseil de la Société des Nations, montrent de façon significative et éloquente la contribution 
efficace et désintéressée qui est apportée par l'Italie à l'œuvre admirable de la Société des Nations" ${ }^{20}$.

Le succès que rencontra l'Institut International du cinématographe éducatif auprès des milieux éducatifs étrangers constituait évidemment une preuve pour le Gouvernement italien du bien-fondé de son initiative. Alfredo Rocco, Président de cet organisme, n'hésitait pas à affirmer que celui-ci était "le plus apprécié parmi les organismes qui dépendaient de la Société des Nations"21.

Il est intéressant de noter qu'à ce combat pour la primauté du pays en matière de cinématographie éducative qu'incarnaient les deux principaux organismes étatiques fascistes sur lesquels nous nous sommes penchés jusqu'alors participait aussi l'industrie cinématographique italienne. Par exemple, au moment où l'Institut International du cinématographe éducatif travaillait à l'unification du format réduit, le Conseil Directeur de l'Association Nationale Fasciste de l'industrie du spectacle se déclara immédiatement prêt à soutenir le développement du format réduit afin que l'Italie restât concurrente sur le domaine international. Il décida en effet de travailler à la coordination des forces industrielles nationales concernées par le cinéma éducatif afin de conquérir le marché mondial en la matière. Voici les résolutions adoptées par son Conseil Directeur en août 1934 :

"Le Conseil considère que la primauté en matière éducative conquise par l'Italie dans le domaine cinématographique - 
surtout à travers l'institution prévoyante qu'est l'Institut National LUCE - doit être renforcée et défendue, et dans ce but les activités industrielles de ce secteur de la production confirment que chaque réalisation dans le domaine de la cinématographie éducative peut aujourd'hui compter sur l'apport exclusif de talent, matériel, main d'œuvre et matières premières italiennes"22.

\section{Les pouvoirs du film éducatif.}

Au-delà de la volonté de marquer la suprématie de l'Italie dans le domaine de la cinématographie éducative, le film éducatif lui-même, considéré de façon matérielle cette fois, était lui aussi utilisé à des fins de propagande à l'étranger. Cet objectif explique que l'Institut National LUCE ait passé des accords avec différentes sociétés afin de régler la distribution de ses propres productions à l'étranger et de répandre ainsi les valeurs fascistes à travers le monde. On attribuait en effet à l'image animée un véritable pouvoir de persuasion ${ }^{23}$. Sa puissance se trouvait accrue du fait qu'un film pouvait toucher simultanément un nombre très important de spectateurs : la communion des foules autour des mêmes images était sensée renforcer l'efficacité de la représentation cinématographique. Voici par exemple ce qu'écrivait Giuseppe Domenico Musso en 1932 à propos du pouvoir du film :

"Le film est l'arme véritable. Cette phrase, dans sa simplicité, synthétise toute la situation. Comme la presse autrefois, la 
cinématographie aujourd'hui est devenue le moyen le plus puissant d'éducation et de propagande. Moyen sûr, rapide, et complètement fascinant"24.

Cette capacité du film à émouvoir les foules explique que l'on ait cherché à l'utiliser dans une perspective de propagande à l'étranger. Dans un article intitulé "Le cinéma comme ambassadeur" et publié dans la revue Cinema en mai 1937, Fritz Olimsky soutenait la thèse selon laquelle cette volonté d'influence culturelle de la part des pays producteurs avait suscité dans de nombreux pays la création d'une industrie cinématographique nationale plutôt que de se contenter d'importer les films produits à l'étranger :

"Nous voulons croire qu'un juste désir de prestige et d'influence culturelle ait fait partie des motifs qui ont conduit de nombreuses nations à initier leur propre production"25.

Le soucis des dirigeants de l'Institut National LUCE de diffuser leurs productions dans différents pays du monde rencontrait la totale approbation du Chef du Gouvernement italien. Voici à titre d'exemple la lettre qu'il envoya le 14 juillet 1925 à Luciano de Feo, alors que ce dernier était encore Directeur de cet Institut :

"La noble tâche de diffusion de la culture et de la science que la "LUCE" accomplit avec ses projections, sa haute contribution à l'élévation morale du peuple, la propagande efficace qu'elle poursuit en Italie et à l'étranger en faisant 
connaître aux étrangers les beautés et les gloires de notre pays et en apportant les vision de la Patrie à ses enfants lointains, méritent toute ma sincère approbation et mes vœux les plus chaleureux et les meilleurs" 26 .

En rendant compte de l'œuvre accomplie par le Gouvernement fasciste en Italie depuis sa prise du pouvoir, les films produits par l'Institut National LUCE constituaient donc un véritable pilier de la propagande fasciste à l'étranger ; d'autant plus que les films éducatifs en question prenaient en compte tous les aspects de la vie italienne et étaient donc emprunts de la philosophie proposée comme modèle par Benito Mussolini. En 1933, Iperato Zanetti exprimait ce sentiment de la façon suivante :

"En relisant les catalogues des films de la cinémathèque LUCE, nous voyons à quel point l'œuvre éducative du Gouvernement National a été étendue à tous les champs de la laborieuse activité italienne pour le renouveau moral et physique de la race"27. $^{27}$.

Pour les mêmes raisons de prestige, le régime fasciste encourageait les projets de films éducatifs et documentaires développés par des maisons de production étrangères et dont le thème concernait l'Italie. Il s'agissait en effet d'une autre manière de mettre en valeur les richesses du pays. Voici comment en septembre 1928, le Ministre de l'Instruction publique expliquait les avantages de telles réalisations à Guido 
Beer, Secrétaire général auprès de la Présidence du Conseil des Ministres :

"Il arrive souvent que de l'étranger, en particulier de l'Amérique du Nord, des individus ou des sociétés fassent des demandes à ce Département pour obtenir l'autorisation de réaliser des bandes cinématographiques sur les monuments d'art ou les ruines antiques des diverses régions du Royaume. De telles demandes sont le plus souvent motivées par des objectifs de propagande culturelle au bénéfice de notre Pays et d'une meilleure connaissance de ses monuments"28.

Ces initiatives étaient évidemment propices à servir les intérêts du régime fasciste : les films ainsi réalisés faisaient en effet connaître à l'étranger le glorieux passé de la Rome antique, les richesses artistiques de l'Italie, et par là même portait l'attention sur le régime qui était au pouvoir à l'époque dans ce même pays.

\section{La cinématographie spectaculaire.}

Ce fut avec un peu de retard par rapport au cinéma éducatif - à partir du milieu des années trente - que le Gouvernement fasciste s'intéressa au cinéma spectaculaire et songea à prendre des mesures afin de le mettre au service de ses propres objectifs. Sa principale ambition était de soumettre cette industrie à l'esprit fasciste qui était sensée organiser l'ensemble de la société italienne ${ }^{29}$. Luigi Chiarini 
retraçait cette prise de conscience dans un ouvrage publié à cette date charnière, en 1935 :

“Aujourd'hui, en réclamant une cinématographie fasciste (et la même chose se produit pour tous les arts), nous voulons que les films expriment l'esprit de notre peuple tel qu'il a été forgé par le Fascisme. Des films, en somme, qui fassent penser, qui approfondissent l'humanité et la moralité des spectateurs, qui lui montrent quels sont les idéaux qui font marcher d'un même pas le peuple italien : ces idéaux de justice sociale dont le Duce a parlé et qui donnent une haute et noble signification à notre volonté de puissance, à notre esprit guerrier et militaire"30.

Cette volonté de prendre en main l'industrie cinématographique spectaculaire se traduisit par un certain nombre de mesures. La première fut la création en 1934 d'un organe politique spécifique, la Direction générale pour la cinématographie, organisée au sein du sous-secrétariat pour la Presse et la propagande et placée sous la direction de Luigi Freddi. Ce dernier se lança dans de grands projets dont les plus représentatifs furent l'ouverture du Centre expérimental de cinématographie ${ }^{31}$, la constitution de l'E.N.I.C. ${ }^{32}$, et enfin la construction des studios de Cinecittà.

Comme pour la cinématographie éducative, le Gouvernement italien envisageait cette œuvre de refonte de l'industrie cinématographique comme un moyen de valoriser l'image de l'Italie à l'étranger et la proposer comme un modèle 
à suivre. Voici à ce propos un extrait d'un article intitulé “L'Italie fait école”, publié dans la revue Cinema en juillet 1936 :

"La mise en place pratique du contrôle de l'État sur la cinématographie en Italie n'a pas manqué d'attirer l'attention des autres pays sur ce que l'on avait déjà réalisé chez nous. Et ce qui ne se serait jamais fait de par leur propre initiative est en cours de réalisation aujourd'hui à l'étranger, par imitation de l'œuvre italienne"33.

La nature de cet objectif poursuivi par le Gouvernement italien fut récemment confirmé par le fils du Duce lui-même, Vittorio Mussolini, au cours d'une interview qu'il accorda en 1993 à Jean-Christophe Rosé. Pour lui, son père aspirait à retrouver la gloire internationale que connaissait le cinéma italien avant la première guerre mondiale :

"Il [Benito Mussolini] voulait d'abord faire de l'industrie cinématographique italienne une industrie florissante. Le cinéma italien avait été connu dans le monde entier à l'époque de la première guerre mondiale. A l'époque des films genre péplum, comme Cabiria, ou à la D'Annunzio. Disons, entre 1910 et 1920. Il n'était peut-être pas le premier du monde, mais il avait été pris comme modèle, même par les américains"34.

Ainsi, au milieu des années trente, les efforts poursuivis en matière de cinématographie éducative s'étendirent à la 
cinématographie spectaculaire, dans l'espoir de faire de l'Italie une référence mondiale et un modèle dans l'univers du film.

Cet aspect de la politique extérieure fasciste italienne mis ici en évidence par rapport au cinéma - et plus spécifiquement au cinéma éducatif - ne constituait qu'un aspect particulier du projet politique global du régime fasciste dans sa volonté de soigner son image à l'étranger. Si la sphère culturelle représentait une part importante de ce programme, le cinéma occupait lui-même une place privilégiée dans la mesure où il constituait un symbole de modernité.

Les résultats qu'obtint l'Italie dans cette perspective furent patents : ses efforts portèrent leurs fruits puisque ce pays s'entoura à l'étranger d'une véritable considération auprès des défenseurs de la cinématographie. Il est d'ailleurs intéressant de noter que l'évolution de la politique extérieure fasciste à partir de la campagne éthiopienne ne suffit pas à effacer cette auréole dont s'était entourée l'Italie en matière culturelle. En effet, après 1935 Benito Mussolini changea totalement de stratégie : il abandonna la politique de séduction qui avait caractérisé jusque là son rapport aux démocraties et se positionna clairement comme ennemis de l'ordre mondial établi. Le rapprochement progressif de l'Italie avec Adolf Hitler à partir de cette date ne fit que conforter cette nouvelle option politique. 
A partir de ce moment là, la perception du régime italien à l'étranger fut marquée par un double mouvement : une condamnation catégorique de ses choix de politique extérieure d'une part, mais doublée d'une admiration persistante pour sa politique intérieure - tout au moins pour ceux qui la manifestait avant les événements éthiopiens - d'autre part. Le même phénomène se manifesta au regard de la cinématographie : dans la seconde partie des années trente, nombre d'étrangers qui ne manifestaient aucune sympathie particulière pour les régimes autoritaires d'une manière générale continuèrent à saluer l'heureux développement de la cinématographie italienne.

Cet extrait d'un article écrit par G. Michel Coissac en mai 1936 était particulièrement significatif quant à la constance de l'enthousiasme des milieux éducatifs français pour les réalisations mussoliniennes en matière de cinématographie dans la seconde moitié des années trente :

"Sans nous occuper outre mesure des questions de régime qui entrent dans le domaine de la liberté individuelle, nous devons nous rendre à l'évidence. Personne ne peut nier qu'en dix ans l'Italie a réalisé des prodiges étonnant d'organisation et de redressement. Que ce soit le fait de la volonté, de l'énergie et de la clairvoyance d'un homme supérieur ; que ce soit l'œuvre du Duce, plutôt que d'un Parlement désormais supprimé, il importe peu pour les gens sans parti pris jugeant les actes aux résultats ; or ces derniers sont patents en Italie. (...) Reconnaissons que chez nos voisins et amis, le cinéma est 
devenu plus et mieux qu'une industrie, une force nationale appliquée à l'enseignement, à l'éducation, aux loisirs ouvriers, au tourisme, à la propagande.

Sans doute l'animateur par excellence est le Chef du Gouvernement, M. Mussolini ; mais nous avons la certitude qu'il a le souci de choisir des collaborateurs compétents et, pour chaque département, des autorités éprouvées, ce qui n’a malheureusement pas lieu en France. Il ne faut donc pas chercher ailleurs la cause de la supériorité de l'Italie dans la civilisation latine" 35 .

Nous conclurons par ces quelques remarques cet aperçu des éléments qui contribuèrent à conférer une certaine aura à la politique intérieure fasciste à l'étranger, et le rôle particulier que joua la cinématographie dans ce processus. Il ne s'agit évidemment que de quelques pistes mais qui offrent un regard différents sur les différents organismes cinématographiques constitués en Italie au cours des années vingt et trente. 
${ }^{1}$ Pour davantage d'informations sur la politique extérieure fasciste, on pourra se reporter aux ouvrages suivants :

- Egidio Reale, Carlo Sforza, La politique fasciste et la Société des Nations, Paris, s.e., 1932.

- Benito Mussolini, L'Italia fascista davanti al mondo, Florence-Rome, La Fenice, Le idee e la storia, 1983, 127 p.

- Richard J.B. Bosworth, Sergio Romani (sous la direction de), La politica estera italiana, 1860-1985, Bologne, Il mulino, 1991, 360 p.

- Carlo M. Santoro, La politica estera di una media potenza, Bologne, Studi e ricerche, $\mathrm{n}^{\circ} 287,1991,351 \mathrm{p}$.

${ }^{2}$ A propos de la politique culturelle fasciste, on pourra consulter :

- Gabriele Turi, Il fascismo e il consenso degli intellettuali, Bologne, Il mulino, 1980, $394 \mathrm{p}$.

- Aspects de la culture italienne sous le fascisme (Actes du colloque de Florence, 14-15 décembre 1979), Grenoble, Publication de l'Université de Grenoble, 1982, 245 p.

- Emilio Gentile, Il culto del littorio. La sacralizzazione della politica nell"Itaila fascista, Rome-Bari, Biblioteca Universale Laterza, 1995, 326 p.

${ }^{3}$ Syndicat pour l'Instruction cinématographique.

${ }^{4}$ L'Union Cinématographique Éducative.

${ }^{5}$ Sur la naissance de l'Institut National LUCE, on pourra consulter :

- L'Istituto Nazionale LUCE, dans Rivista del cinematografo, Milan, n6-7, juin-juillet 1928, p. 85-88. 
- Alessandro Sardi, Cinque anni di vita dell'Istituto Nazionale "Luce", Rome, Grafia, 1929, 122 p.

- Lo schermo, Rome, juillet 1936, 64 p (numéro spécial sur l'Institut National LUCE).

- Origine, organizzazione e attività dell'Istituto Nazionale LUCE, Rome, Istituto poligrafico dello stato, 1934, $62 \mathrm{p}$.

- Ipotesi per un catalogo, Rome, Istituto LUCE, Italnoleggio cinematografico, 1990, $143 \mathrm{p}$.

${ }^{6}$ G. Michel Coissac, L'action du cinéma en Italie, dans Le Cinéopse, Paris, mai 1936, p. 89-91.

7 Jehan de Vimbelle, Chronique du cinéma éducateur, dans Le Cinéopse, Paris, octobre 1927, p 864.

${ }^{8}$ Richard S. Lambert, L'Institut Britannique du cinématographe, dans Revue Internationale du cinéma éducateur, Rome, mai 1933, p. 343-349.

${ }^{9}$ Luciano de Feo, Come nacque l'Istituto Nazionale LUCE, in Lo Schermo, Rome, juillet 1936, p 20.

(Texte original : "Ma una cosa è certa. Che il Regime Fasciste seppe, per il primo, ed opera del suo Capo, limpidamente comprendere e antivedere quello che potesse significare, per il popolo, l'arma magnifica e terribile dello schermo, atta a creare e forgiare gli spiriti, o a deprimerli, a volontà, a seconda della mentalità di chi intenda usarla ed a seconda che, allo schermo, si voglia dare una veste di mercantilismo puro od un senso di alta spiritualità. Parecchi altri Istituti del genere sont sorti nel mondo. Dopo, non prima. "LUCE" è stata luce per tutti"). 
${ }^{10}$ Proposition italienne pour la création d'un Institut International du cinématographe éducatif à Rome : suite à donner à la décision prise par l'Assemblée au cours de sa huitième session ordinaire, Procès-verbal de la quarante-septième session du Conseil, dans Société des Nations. Journal Officiel, Genève, avril 1928, p. 1450-1451.

${ }^{11}$ Lettre de Filippo Cremonesi au Ministre des Affaires étrangères, 30 juin 1927, Rome, Archivio Centrale dello Stato, Presidenza Consiglio Ministri 1931-1933, 5/1/6893, 2 p.

(Texte original : "La LUCE è lieta e fiera di poter collaborare con ogni sua forza alla vittoriosa affermazione del Nostro Paese nel campo della cinematografia educativa. Non esitiamo a dichiarare che la istituzione che l'E.V. sta studiando ed intende realizzare potrà essere di grande giovamento per il nostro Paese").

12Sur les travaux de l'Institut International du cinématographe éducatif, se reporter à :

- L'activité de l'Institut du cinématographe éducatif (Société des Nations) dans ses publications officielles, Rome, Institut International du cinématographe éducatif, s.d. (1930).

- L'activité de l'Institut International du cinématographe éducatif en 1930. Rapport de Luciano de Feo au Conseil d'administration, Rome, Institut International du cinématographe éducatif, Cahier n.26, s.d. (1930), 44 p. - Institut International du cinématographe éducateur, dans Le nouvel art cinématographique, Brest, janvier 1930, p. 7-9.

- L'Istituto Internazionale per la cinematografia educativa e le industrie del cinema, dans Rivista del cinematografo, Milan, février 1929, p. 45. 
- L'Istituto Internazionale del cinematografo educativo. Discorso del Ministro della Giustizia per la inaugurazione dell'ICE, dans Rivista del cinematografo, Milan, mars 1929, p. 71-73.

${ }^{13}$ La vie de l'Institut, dans Revue Internationale du cinématographe éducatif, Rome, avril 1930, p. 471.

${ }^{14}$ Lettre de Luciano de Feo à Benito Mussolini, 10 septembre 1929, Rome, Archivio Centrale dello Stato, Segreteria Particolare del Duce, Carteggio ordinario, Busta 265, fascicolo 12.501, 1 p.

(Texte original : "Sapendo di far cosa grata all'E.V. ho l'onore di comunicarLe che, durante il mese di agosto, gli articoli e le notizie pubblicate in favore dell'Istituto, in tutti i paesi del mondo, superano il migliaio").

${ }^{15}$ Lettre de Luciano de Feo à Benito Mussolini, 5 novembre 1931, Rome, Archivio dello Stato, Presidenza Consiglio Ministri 1931-1933, 5/1/6893, 1

p.

(Texte original : "Posso garantire a V.E. che nulla sarà trascurato e tutto compiuto perchè l'Istituto - oggi conosciuto ed apprezzato in tutto il mondo - possa svolgere un'opera sempre più grande, degna della Patria che ci comanda di affermare in ogni istante - ovunque e contro tutti - la sua volontà di non essere seconda a nessuno").

${ }^{16}$ Lettre de Luciano de Feo à Benito Mussolini, 10 septembre 1929, op. cit. (Texte original : "Prometto a V.E. che la Rivista arriverà ad un quantitativo mensile di 30.000 esemplari diffusi in 52 paesi. In tal modo l'ordine datomi da V.E. di creare a Roma - nell'Italia Fascistissima, un centro mondiale di 
studio di tutti i problemi (...) del cinema, sarà assolto con la gioia immensa di servire sempre (...) l'E.V. ed il Fascismo").

${ }^{17}$ Discours retracé dans l'article suivant : Il festival del cinema, dans Il gazzettino, 10 août 1932.

(Texte original : "L'oratore sata quindi in nostro Governo Nazionale e il Duce che questo problema hanno compreso, e l'Italia, che diede i primi film veramente artistici, quale l'indimenticabile Cabiria di D'Annunzio, porta ora un magnifico contributo a quest'arte che tornerà ringagliardita dal convegno veneziano. L'oratore conclude : "Porgo il mio rispettoso omaggio a S.E. Benito Mussolini che da noi francesi è considerato alla stregua di Cesare e Napoleone").

${ }^{18}$ Circulaire n²34630-C signée par Benito Mussolini concernant le "Congresso internazionale del cinema di insegnamento e di educazione", (20 novembre 1933), Rome, Archivio dello Stato, Presidenza Consiglio Ministri 1931-1933, 14/3/8838, 3 p.

(Texte original : "L'iniziativa ha il pieno apporggio del R. Governo che vede con favore quanto possa contribuire ad accrescere il prestigio e l'importanza dell'Istituto, ad allargarne la sfera d'azione in un campo nel quale l'Italia per prima ha indicato un orientamento costruttivo e nel quale ha realizzato notevoli progressi").

${ }^{19}$ Benito Mussolini, L'Italia nel mondo, Atti parlamentari della Camera dei senatori, discussioni, dans L'Italia fascista davanti al mondo, FlorenceRome, La Fenice, Le idee e la storia, 1983. 
(Texte original : "Questi sono "fatti" eloquenti più di qualsiasi discorso a caratterizzare l'atteggiamento del Governo fascista verso la Lega delle Nazioni").

${ }^{20}$ Louis-Dop, Le rôle et la mission de l'Institut International du cinématographe éducatif dans le cadre des organisations internationales officielles, dans Revue Internationale du cinématographe éducateur, Rome, juillet 1929.

${ }^{21}$ Lettre d'Alfredo Rocco à Benito Mussolini, 3 novembre 1933, Rome, Archivio Centrale dello Stato, Presidenza Consiglio Ministri 1931-1933, 5/1/6893, 1 p.

(Texte original : "(...) il più apprezzato fra quanti dipendono dalla Società delle Nazioni").

${ }^{22}$ Lettre du Directeur des services économiques de la Confédération Générale Fasciste de l'Industrie du spectacle à la Présidence du Conseil des Ministres, 16 août 1934, Rome, Archivio Centrale dello Stato, Presidenza Consiglio Ministri 1934-1936, 3/1.7/2100, p 5.

(Texte original : "Il Consiglio ritiene che il primato educativo nel mondo conquistato nel campo cinematografico dall'Italia, soprattutto attraverso la provvida istituzione dell'Istituto Nazionale LUCE, dabba essere potenziato e difeso, ed a tale scopo le attività industriali di questo settore della produzione confermano che ogni realizzazione nel campo della cinematofrafia educativa, può oggi fare assegnamento sull'esclusivo apporte di ingegno, materiale, mano d'opera e materie prime italiane"). 
${ }^{23}$ Sur les réflexions relatives au pouvoir de l'image à l'époque, on pourra consulter :

- Leo Lionni, Ambiente razionale didattico, Rome, Edizioni della confederazione fascista degli industriali, 1937, 15 p.

- Antonio Todisco, L'organizzazione della propaganda rurale e cinematografica nell'insegnamento scolastico, Rome, Edizioni della confederazione fascista degli industriali, 1937, 12 p.

- Vinicio Araldi, Cinema, arma del tempo nostro, Milan, La Prora, 1939, 200 p.

${ }^{24}$ Giuseppe Domenico Musso, Le possibilità della cinematografia come mezzo di propaganda e di educazione, Rome, Luzzetti, 1932.

(Texte original : "Il film è la vera arma. Questa frase nella sua semplicità, sintetizza tutta la situazione. Come la stampa, una volta, la cinematografia, oggì, è diventata il mezzo più potente di educazione e di propaganda. Mezzo sicuro, rapido, ed intensamente affascinante").

${ }^{25}$ Fritz Olimsky, Il cinema come ambasciatore, dans Cinema, Rome, mai 1937.

(Texte original : "Vogliamo credere che un giusto desiderio di prestigio e d'influenza culturale sia stato tra i motivo che hanno indotto molte nazioni ad iniziare una produzione propria").

${ }^{26}$ Lettre de Benito Mussolini à Luciano de Feo du 14 juillet 1925, citée dans : G. Michel Coissac, L'action du cinéma en Italie, dans Le Cinéopse, Paris, mai 1936, p. 89-91. 
${ }^{27}$ Iperato Zanetti, La cinematografia e la scuola fascista. La cinematografia maestra di vita, Trieste, s.e., 1933, p 3.

(Texte original : "Rileggendo gli elenchi dei film della cineteca di LUCE, vediamo come l'opera educativa del Governo Nazionale sia stata estesa a tutti i campi dell'operosa attività italiana per il rinnovamento morale e fisico della stirpe").

${ }^{28}$ Lettre du Ministre de l'Instruction publique à Guido Beer, 25 septembre 1928, Rome, Archivio dello Stato, Presidenza Consiglio Ministri 1928-30, 5/2/4341, 2 p.

(Texte original : "Accade di frequente che da parte di stranieri, singoli privati o società, specialemente del Nord-America, vengano avanzate istanze a questo Dicastero per ottenere l'autorizzazione a trarre pellicole cinematografiche da monumenti d'arte o scavi di antichità nelle diverse regioni del Regno. Tale domande sono per solito motivate da fini di propaganda colturale a vantaggio del nostro Paese e della migliore conoscenza dei suoi monumenti").

${ }^{29}$ Sur l'histoire de la politique cinématographique fasciste, on pourra entre autres consulter :

- Luigi Chiarini, Cinematografo, Rome, Cremonese, 1935, 118 p.

- Luigi Freddi, Il cinema, Rome, L'Arnia, 1949, 2 volumes, 450 p, 550 p.

- Mino Argentieri, L'occhio del regime. Informazione e propaganda nel cinema del fascismo, Florence, Vallecchi editore, 1979, 202 p.

- Jean A. Gili, Le cinéma italien à l'ombre des faisceaux (1922-1945), Nice, Institut Jean Vigo, 1990, 319 p.

- Renzo Renzi (sous la direction de), Il cinema dei dittatori. Mussolini, Stalin, Hitler, Bologne, Grafis edizioni, 1992, 300 p. 
${ }^{30}$ Luigi Chiarini, Cinematografo, Rome, Cremonese, 1935, 118 p.

(Texte original : "Oggi chiedendo una cinematografia fascista (e lo stesso avviene per tutte le arti) chiediamo film che esprimano lo spirito del nostro popolo così come è stato forgiato dal Fascismo. Dei film, insomma, che facciano pensare, che approfondiscano l'umanità e la moralità degli spettatori, che li accendano per quelli ideali che oggi fan marciare compatto il popolo italiano : quegli ideali di giustizia sociale di cui il Duce ha parlato e che danno un alto e nobilissimo significato alla nostra volontà di potenza, al nostro spirito guerriero e militare").

${ }^{31}$ École destinée à former les futurs professionnels de l'industrie cinématographique ; elle ouvrit le 18 novembre 1935 et sa direction fut confiée à Luigi Chiarini.

32 "Office national pour les industries cinématographiques", organe destiné à venir en aide aux nombreuses sociétés cinématographiques (production, distribution, exploitation) atteintes par la crise économique.

${ }^{33}$ L'Italia fa scuola, dans Cinema, Rome, 25 juillet 1936, p 72.

(Texte original : "La pratica attuazione del controllo statale sulla cinematografia in Italia non ha mancato di attirare l'attenzione degli altri Paesi su quanto si era già realizzato da noi. E quello che non si sarebbe mai fatto di spontanea volontà si va facendo oggi all'estero con criterio imitativo dell'opera italiana").

${ }^{34}$ Vittorio Mussolini, cinéma et fascisme, Documentaire de Jean-Christophe Rosé, 65 mn, 1993, couleur. 
${ }^{35}$ G. Michel Coissac, L'action du cinéma en Italie, dans Le Cinéopse (Paris), mai 1936, p 90. 